\title{
Estrutura de uma comunidade arbórea adulta na Amazônia e o desafio de uso sustentável de espécies florestais comerciais
}

O Brasil tem a maior biodiversidade de fauna e flora do Planeta, com grande potencial e dificuldades para o manejo dos seus recursos naturais. Neste estudo, fo analisada a estrutura de 84 hectares de floresta natural, na Fazenda Rio Capim, pertencente à empresa florestal Cikel Brasil Verde Ltda. Foram registradas 1435 árvores com DAP $=45 \mathrm{~cm}$, distribuídas em 65 espécies, 54 gêneros e 22 famílias. As espécies mais importantes foram: Eschweilera sp. com Índice Valor de Cobertura (IVC) de 32,62, seguida por Manilkara amazônica (IVC = 19,36). A espécie Hymenaea courbaril, apresentou o maior volume $(5,115$ m3.ha-1). As espécies Manilkara huberi, Goupia glabra e Lecythis usitata também apresentaram expressivos IVC's e volume. As espécies Brosimum parinarioides, Protium puncticulatum, Anadenanthera colubrina e Lecythis lurida completam a lista das mais importantes na área estudada. A maioria das espécies tem sua madeira comercializada no mercado doméstico, mas algumas atingem o mercado internacional. Outras espécies apresentam grande importância na produção de produtos não-madeireiros como, fitoterápicos, fibras e frutos. As recomendações aqui apresentadas podem ser aplicadas em outras florestas tropicais com estrutura semelhante à da floresta estudada neste trabalho.

\section{Structure of an old tree community in the Amazon and the challenge of sustainable use of commercial tree species}

Brazil has the largest biodiversity of fauna and flora of the world. This brings a great potential and challenges for forest management in the country. In this study the structure of 84 hectares of natural forest was analyzed, in the Rio Capim Farm that belongs to the forestry company CIKEL Brasil Verde Ltd. It was registered a number of 1435 trees with $\mathrm{dbh}=45 \mathrm{~cm}$, distributed in 65 species, 54 genera and 22 families. The most important species were: Eschweilera sp. with a Coverage Index Value (CIV) of 32.62, followed by Manilkara amazônica (CIV = 19.36). The species Hymenaea courbaril presented the biggest volume $(5,115$ m3.ha-1). The species Manilkara huberi, Goupia glabra, and Lecythis usitata also had high CIVs and volumes. The species Brosimum parinarioides, Protium puncticulatum, Anadenanthera colubrina, and Lecythis lurida complete the list of the most important species in the studied area. Most of the species are traded in the domestic market, but part of these species reaches the international market. Other species have great importance for producing non-timber forest products such as medicines for phytotherapy, fibers, and fruits. The recommendations presented here can be applied in other tropical forests with similar structure of the forest studied in this work.

Keywords: Floristic composition; Use of wood; Non-timber forest products; Amazonian natural forest; Forest management.

Topic: Ciências Florestais

Reviewed anonymously in the process of blind peer.
Received: 04/08/2020

Approved: 18/09/2020
Klewton Adriano Oliveira Pinheiro (io)

Instituto Federal do Pará, Brasil

http://lattes.cnpq.br/8139678842009696

http://orcid.org/0000-0003-2696-4249

klewton.pinheiro@gmail.com

João Olegário Pereira de Carvalho (iD

Universidade Federal Rural da Amazônia, Brasil

http://lattes.cnpq.br/0989611785962681

http://orcid.org/0000-0001-9396-2417

olegario.carvalho@gmail.com

Gustavo Schwartz (iD)

Empresa Brasileira de Pesquisa Agropecuária, Brasil

http://lattes.cnpq.br/0774787368316223

http://orcid.org/0000-0002-1717-4491

gustavo.schwartz@embrapa.com

\author{
Luciana Maria de Barros Francez (iD) \\ Universidade Federal Rural da Amazônia, Brasil \\ http://lattes.cnpq.br/5872429121209613 \\ http://orcid.org/0000-0001-6688-5639 \\ lucianafrancez@yahoo.com.br \\ Beatriz Quanz (iD) \\ Universidade Federal Rural da Amazônia, Brasi \\ http://lattes.cnpq.br/8533879167092765 \\ http://orcid.org/0000-0002-3608-3739 \\ biaquanz@yahoo.com.br \\ Francimary da Silva Carneiro (iD \\ Universidade Federal Rural da Amazônia, Brasil \\ http://lattes.cnpq.br/8657235544233319 \\ http://orcid.org/0000-0002-1693-8779 \\ francimarycarneiro@gmail.com
}

Referencing this:

PINHEIRO, K. A. O.; CARVALHO, J. O. P.; SCHWARTZ, G.; FRANCEZ, L. M. B.; QUANZ, B.; CARNEIRO, F. S.. Estrutura de uma comunidade arbórea adulta na Amazônia e o desafio de uso sustentável de espécies florestais comerciais. Revista Ibero Americana de Ciências Ambientais, v.11, n.5, p.63-73, 2020. DOI:

http://doi.org/10.6008/CBPC2179-6858.2020.005.0007 


\section{INTRODUÇÃO}

O setor florestal madeireiro está entre os mais importantes na economia da Amazônia brasileira. Em 2008, apenas o estado do Pará, foi responsável por movimentar um valor de US\$ 4,46 bilhões e gerar 30.481 empregos, o que representou 9,6\% do PIB e 3,6\% do emprego total no estado (SANTANA et al., 2012).

Apesar dos efeitos positivos à economia local, a exploração madeireira em florestas tropicais nativas não vem sendo sustentável. Isto leva a diminuição de áreas cobertas por florestas e consequente perda de seus bens e serviços ambientais (MYERS, 1993). No contexto da Amazônia brasileira, aproximadamente 70\% da madeira produzida na região é ilegal (LAWSON et al., 2010). Esta madeira é extraída da floresta sem nenhum planejamento, o que provoca danos ás espécies exploradas e a floresta remanescente (PINTO, et al. 2002; SABOGAL, 2006; FRANCEZ, et al., 2009). Entre as alternativas para a produção sustentável de madeira na Amazônia existe a exploração de impacto reduzido ou exploração de baixo impacto. Este tipo de exploração florestal é menos prejudicial ás espécies exploradas e a floresta remanescente, já que através da intervenção são retirados de dois a três indivíduos por hectare (BRASIL, 2006; MAUÉS, 2006; DYKSTRA, 2012; SCHWARTZ et al., 2012).

A busca de formas sustentáveis para a exploração de florestas nativas é essencial para a manutenção da diversidade de espécies em seus ecossistemas naturais (ALVES et al., 2008). Nesses ecossistemas, os processos ecológicos são complexos e dinâmicos. Assim quaisquer intervenções para fins de produção de madeira ou mesmo de produtos não madeireiros demandam necessariamente conhecimento prévio sobre funcionalidade das espécies e das florestas a serem exploradas de modo a alcançar-se um manejo sustentável (BENTES-GAMA, 2000).

Outro aspecto importante para se produzir resultados mais eficientes no manejo florestal é o conhecimento das características e propriedades das diferentes comunidades florestais, para evitar alterações significativas na composição florística e estrutura da floresta (OLIVER et al., 1996). O conhecimento detalhado da composição florística e da estrutura da floresta manejada possibilita a realização de estudos fitossociológicos para quantificar o potencial madeireiro de espécies comerciais, além de prever os tratamentos silviculturais mais aconselháveis a serem aplicados na floresta manejada (VALE, 1972; RODRIGUEZ, 1998).

O presente estudo tem como objetivo analisar a composição florística e a estrutura horizontal de 84 ha de floresta natural na Fazenda Rio Capim, município de Paragominas/PA, considerando espécies comerciais. Os resultados deste trabalho podem ter aplicação direta no manejo e conservação de outras florestas tropicais com estruturas semelhantes à floresta abordada neste estudo.

\section{METODOLOGIA}

\section{Área de estudo}

O trabalho foi realizado na Fazenda Rio Capim, que possui área de 140.658 ha, pertencente à empresa florestal Cikel Brasil Verde S/A (FRANCEZ et al., 2009), localizada no município de Paragominas, 
distante cerca de $320 \mathrm{~km}$ de Belém (Figura 1). A área é caracterizada por possuir períodos de elevados índices de chuva, $250 \mathrm{~mm}$ mensais, alternado por períodos secos (LEAL, 2000). A estação chuvosa se estende entre novembro/dezembro a março/abril com precipitação pluviométrica anual de 1.800 a $2.100 \mathrm{~mm}$. A temperatura média anual é de $27^{\circ} \mathrm{C}$ e a umidade relativa do ar é alta, com valores entre 80 e $85 \%$. 0 clima dominante na região, segundo a classificação de Köeppen, é do tipo 'Aw'.
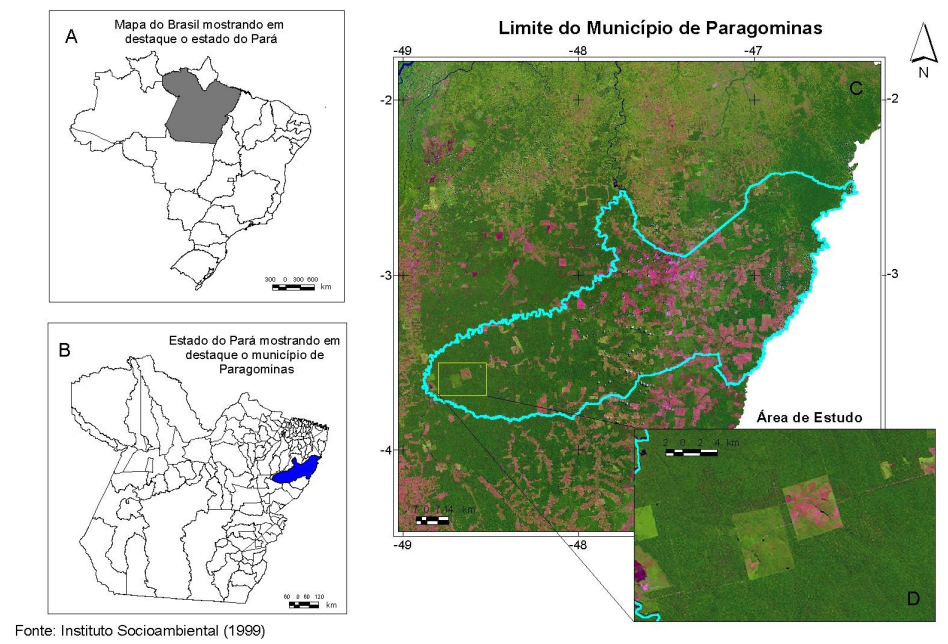

Figura 1: Localização da área de estudo na fazenda Rio Capim (C), município de Paragominas (B), estado do Pará, Brasil (A).

A área está sobre rochas sedimentares do Cretáceo Superior pertencentes à Bacia do Grajaú. A topografia é plana (planícies aluviais) a suavemente ondulada, com altitude média de $20 \mathrm{~m}$ em relação ao nível do mar (WATRIN et al., 1992). O solo dominante na região é o Latossolo Amarelo de textura média e muito argilosa (SILVA, 1997; MORAIS-CRUIA et al., 1999).

A área da fazenda está nas bacias do rio Capim e do rio Surubijú. Outros rios de menor porte drenam a área, tais como os rios Cauaxí, Candiru-Açu, Água Boa, Timbó-Açu e Matamatá (WATRIN et al., 1992). A vegetação na fazenda é bem diversificada, de acordo com a classificação de Veloso (1991) predominam a Floresta Ombrófila Densa e a Floresta Ombrófila Aluvial.

\section{Obtenção e análise de dados}

O estudo foi desenvolvido em uma área de 84 hectares de Floresta Ombrófila Densa primária (Unidade de Trabalho no 16 - UT 16, da Unidade de Produção Anual no 06 - UPA 06) do projeto de manejo florestal da fazenda Rio Capim. Foi realizado um inventário pré-exploratório de árvores com diâmetro igual ou superior a $45 \mathrm{~cm}$ das espécies comercializadas na região ou com potencial de mercado.

Para a realização do inventário, foram abertas picadas separadas $50 \mathrm{~m}$ uma das outras. As picadas foram devidamente marcadas com piquetes de $1,5 \mathrm{~m}$ a cada $25 \mathrm{~m}$ em toda a extensão da área amostrada. $\mathrm{A}$ medição das árvores foi feita com fita diamétrica e cada árvore foi devidamente numerada com plaqueta de alumínio e marcada com tinta vermelha.

As espécies foram relacionadas pelo nome local e identificadas pela equipe técnica e com o auxílio de literatura especializada. Para os indivíduos não identificados em campo foi coletado material botânico 
para identificação no Herbário IAN da Embrapa Amazônia Oriental. Determinou-se a abundância, a área basal e o volume das espécies inventariadas. A abundância foi determinada pela relação entre o número de árvores e uma unidade de área (ha); a área basal foi calculada pela soma das áreas transversais das árvores de cada espécie, utilizando a fórmula:

$$
A T=\frac{\pi D A P^{2}}{4}
$$

Onde, AT é a área transversal e DAP é o diâmetro a 1,30m do solo. Como ainda não há uma equação de volume para a área, o cálculo do volume foi feito através da fórmula:

$$
\mathrm{V}=\mathrm{AT} \cdot \mathrm{h} \cdot 0,7
$$

Onde, $v$ = volume, $\mathrm{h}=$ altura comercial da árvore e 0,7 é o fator de forma. Determinou-se também o Índice de Valor de Cobertura (IVC) através da fórmula: IVC = Do+AB, onde, Do e $A B$ são os valores relativos de densidade e dominância, respectivamente. Eles permitem definir o grau de cobertura de cada espécie na comunidade (BENTES-GAMA, 2000; PINHEIRO et al., 2007).

Foram coletados dados secundários, na literatura, em relação às características tecnológicas e usos da madeira das principais espécies, assim como os seus usos não madeireiros. As características e uso da madeira das espécies descritas neste estudo são apresentados de acordo com resultados e recomendações de Loureiro (1979), Sudam (1979), Souza et al. (1997) e Pinheiro et al. (2009).

\section{RESULTADOS E DISCUSSÃO}

\section{Composição florística e abundância de famílias}

Foram registrados 1435 indivíduos com DAP $\geq 45 \mathrm{~cm}$, distribuídos em 65 espécies, 54 gêneros e 22 famílias. A família Leguminosae foi a que apresentou a maior riqueza de espécies (17), distribuídas em 15 gêneros. Apesar de a família Leguminosae ser a mais rica em espécies, ela apresentou um número de indivíduos relativamente menor do que nas famílias Lecythidaceae e Sapotaceae.

Tabela 1: Composição florística, volume $\left(\mathrm{m}^{3} \cdot \mathrm{ha}^{-1}\right)$, área basal ( $\mathrm{AB}$ ) e Abundância (№ árv.) da UT 16 da UPA 06 de 84 ha da Fazenda Rio Capim, considerando árvores com DAP $\geq 45 \mathrm{~cm}$, de espécies de valor comercial ou com potencial para

\begin{tabular}{|c|c|c|c|}
\hline Família/nome comum /Nome científico & $\begin{array}{l}\text { Volume } \\
\left(\mathrm{m}^{3} . \mathrm{ha}^{-1}\right)\end{array}$ & $\begin{array}{l}\text { AB.ha }{ }^{-1} \\
\left(\mathrm{~m}^{2} / \mathrm{ha}\right)\end{array}$ & № Arv.ha-1 \\
\hline \multicolumn{4}{|l|}{ Anacardiaceae } \\
\hline Cajú-açu (Anacardium giganteum Loud. ex Steud.) & 0,547 & 0,059 & 0,119 \\
\hline Muiracatiara (Astronium ulei Marttick) & 1,157 & 0,1 & 0,297 \\
\hline \multicolumn{4}{|l|}{ Araliaceae } \\
\hline Morototó (Schefflera morototoni (Aubl.) Decne \& Planch) & 0,118 & 0,014 & 0,047 \\
\hline \multicolumn{4}{|l|}{ Bignoniaceae } \\
\hline Ipê-amarelo (Tabebuia ochracea (Cham.) Standl.) & 0,013 & 0,001 & 0,011 \\
\hline Ipê-roxo (Tabebuia serratifolia (Vahl) Nichols.) & 1,332 & 0,115 & 0,083 \\
\hline Parapará (Jacaranda copaia (Aubl.) D. Don) & 0,256 & 0,036 & 0,154 \\
\hline \multicolumn{4}{|l|}{ Bombacaceae } \\
\hline Sumaúma (Ceiba pentandra K. Scum.) & 0,656 & 0,089 & 0,285 \\
\hline \multicolumn{4}{|l|}{ Boraginaceae } \\
\hline Freijó (Cordia sp.) & 0,037 & 0,004 & 0,011 \\
\hline Freijó-branco (Cordia alliodora (Ruiz \& Pav.) Cham. Ex. A. DC.) & 0,052 & 0,008 & 0,047 \\
\hline Freijó-cinza (Cordia goeldiana A. DC. Huber) & 0,54 & 0,062 & 0,214 \\
\hline \multicolumn{4}{|l|}{ Burseraceae } \\
\hline Amesclão (Trattinickia burseraefolia (Mart) Willd.) & 0,636 & 0,082 & 0,19 \\
\hline Breu-vermelho (Protium puncticulatum Macbride) & 1,514 & 0,253 & 1 \\
\hline
\end{tabular}
serem comercializadas. 


\section{Caryocaraceae}

Piquiá (Caryocar microcarpum Ducke)

Piquiarana (Caryocar glabrum (Aubl.) Pers.)

Celastraceae

Cupiúba (Goupia glabra Aubl.)

Chrysobalanaceae

Oiticica (Licania sclerophylla (Mart. Ex Hook.) Fritsch.)

\section{Combretaceae}

Tanimbuca (Buchenavia grandis Ducke)

Humiriaceae

Uxi (Endopleura uchi (Huber) Cuatrec.)

Lauraceae

Louro-abacate (Aniba burchellii Kosterm.)

Louro-amarelo (Aniba terminalis Ducke)

Louro-canela (Ocotea fragantissima Ducke)

Louro-preto (Ocotea baturitensis (Nees) Kosterm.)

Louro-rajado (Ocotea cymbarum H.B.K.)

Louro-vermelho (Nectandra rubra (Mez.) C.K. Allen)

\section{Lecythidaceae}

Jarana (Lecythis lurida (Miers) Mori)

Matamatá (Eschweilera sp.)

Tauari (Couratari oblongifolia Ducke \& R. Knuth)

\section{Leguminosae}

Angelim-amargoso (Vatairea sericea Ducke)

Angelim-pedra (Dinizia excelsa Ducke)

Angelim-rajado (Pithecelobium racemosum Ducke)

Angico (Piptadenia suaveolens Miq)

Copaíba (Copaifera multijuga Hayne)

Cumaru (Dipteryx odorata (Aubl.) Willd.)

Fava-atanã (Parkia gigantocarpa Ducke)

Muirarema (Parkia pendula (Willd.) Benth. ex Walp.)

Fava-bolota (Parkia nítida Ducke)

Faveira-branca (Parkia multijuga Benth)

Jatobá (Hymenaea courbaril L.)

Jatobá-curuba (Hymenaea sp.)

Melancieira (Alexa grandiflora Ducke)

Orelha-de-macaco (Enterolobium sp.)

Roxinho (Peltogyne paradoxa Ducke)

Sucupira (Diplotropis sp.)

Sucupira-folha-fina (Diplotropis purpurea (Rich.))

Sucupira-preta (Bowdichia nitida Spruce)

Sucupira-tento (Abarema jupumba (Willd.) Britton \& Killip)

Tamboril (Enterolobium maximum Ducke.)

Taxirana (Sclerolobium paraense Huber)

Meliaceae

Andiroba (Carapa guianensis Aubl.)

Cedro (Cedrela odorata L.)

\section{Moraceae}

Amapá-doce (Brosimum parinarioides Ducke)

Mururé (Brosimum obovata Ducke)

Tatajuba (Bagassa guianensis Aubl.)

Myristicaceae

Virola (Virola melinonii W. A. Rodrigues)

Opiliaceae

Pau-marfim (Agonandra brasiliensis (Miers))

Rubiaceae

Escorrega-macaco (Capirona huberiana Ducke)

Rutaceae

Pau-amarelo (Euxylophora paraensis Huber)

Sapotaceae

Goiabão (Pouteria pachycarpa Pires)

Guajará-bolacha (Pouteria sp.)

Maçaranduba (Manilkara huberi (Ducke) Standley.)

Maparajuba (Manilkara amazônica Stand.)

Simarubaceae

Marupá (Simaruba amara Planch.)
Sapucaia (Lecythis usitata Miers)

\begin{tabular}{|c|c|}
\hline 0,829 & 0,128 \\
\hline 0,916 & 0,135 \\
\hline 2,577 & 0,41 \\
\hline 0,014 & 0,002 \\
\hline 0,344 & 0,05 \\
\hline 0,051 & 0,007 \\
\hline 0,61 & 0,094 \\
\hline 0,015 & 0,002 \\
\hline 0,102 & 0,017 \\
\hline 0,862 & 0,132 \\
\hline 0,127 & 0,015 \\
\hline 0,408 & 0,062 \\
\hline 1,057 & 0,11 \\
\hline 4,814 & 0,807 \\
\hline 1,923 & 0,265 \\
\hline 0,381 & 0,053 \\
\hline 0,275 & 0,036 \\
\hline 0,252 & 0,029 \\
\hline 0,031 & 0,004 \\
\hline 1,514 & 0,253 \\
\hline 0,179 & 0,021 \\
\hline 0,373 & 0,055 \\
\hline 0,277 & 0,038 \\
\hline 0,379 & 0,065 \\
\hline 0,024 & 0,003 \\
\hline 0,576 & 0,071 \\
\hline 5,115 & 0,438 \\
\hline 0,048 & 0,006 \\
\hline 0,67 & 0,098 \\
\hline 0,217 & 0,039 \\
\hline 0,516 & 0,061 \\
\hline 0,016 & 0,002 \\
\hline 0,013 & 0,002 \\
\hline 0,309 & 0,028 \\
\hline 0,229 & 0,037 \\
\hline 0,02 & 0,002 \\
\hline 0,167 & 0,024 \\
\hline 0,617 & 0,099 \\
\hline 0,08 & 0,009 \\
\hline 0,432 & 0,049 \\
\hline 0,309 & 0,043 \\
\hline 0,557 & 0,07 \\
\hline 0,33 & 0,044 \\
\hline 0,943 & 0,123 \\
\hline 0,278 & 0,04 \\
\hline 0,881 & 0,156 \\
\hline 0,403 & 0,054 \\
\hline 0,529 & 0,066 \\
\hline 2,721 & 0,314 \\
\hline 4,724 & 0,584 \\
\hline 0,147 & 0,016 \\
\hline
\end{tabular}




\section{Sterculiaceae}

Axixá (Sterculia pilosa Ducke)

0,587

0,095

0,404

Vochysiaceae

Mandioqueira (Qualea lancifolia Ducke)

0,287

46,913

0,043

0,071

Total

Whitmore (1990) comenta que a família Leguminosae destaca-se por apresentar alta dominância de espécies em florestas neotropicais, o que foi corroborado por este e outros trabalhos (COSTA et al. 2002; FRANCEZ et al. 2007; PINHEIRO et al. 2007; FRANCEZ, et al. 2009). Silva et al. (2008), encontraram 38 espécies de leguminosas (23\% do total) na Floresta Nacional do Tapajós (região de Santarém/PA) enquanto que em área próxima (Curua-Una/Pará), Barros (2000) levantou 14 espécies (10,45\% do total) de leguminosas. Em ambos os estudos, as amostras incluíram indivíduos com DAP $\geq 45 \mathrm{~cm}$. Ainda na mesma região (Belterra) em área de capoeira alta com aproximadamente de 50 anos de regeneração, Carvalho et al. (1986) também registraram a família Leguminosae como a mais importante na composição florística, tendo 27 espécies em 20 gêneros ( $20 \%$ do total amostrado).

Neste trabalho as famílias mais abundantes quanto ao número de indivíduos foram: Lecythidaceae (355), Leguminosae (282), Sapotaceae (236), Burseraceae (100), Lauraceae (80) e Celastraceae (68). Estes resultados são compatíveis com vários estudos realizados na Amazônia como, por exemplo, Salomão (1991) que em uma vegetação de mata de terra firme em Marabá-PA, registrou a família Lecythidaceae como a mais importante. Silva et al. (1992) também registraram maior importância para a família Lecythidaceae em levantamento florístico na bacia do Juruá. A mesma tendência foi observada por Merona et al. (1992) próximo a Manaus, onde registraram as famílias Lecythidaceae, Leguminosae, Sapotaceae e Burseraceae como as mais abundantes em indivíduos e espécies. Por outro lado, Milliken (1998) encontrou a família Leguminosae seguida por Lecythidaceae e Sapotaceae como as mais abundantes em área de terra firme perto da aldeia de Maré, na margem direita do Rio Camanaú 200 km de Manaus no estado do Amazonas.

Das 65 espécies registradas (Tabela 1), 16 (24,6\% do total) apresentaram baixa abundância, com menos de cinco indivíduos. Segundo Kageyama et al. (2000), as espécies podem ser divididas em três grupos, de acordo com sua densidade, em ecossistemas não perturbados: a) raras, com menos de um indivíduo por hectare; b) intermediárias, com um indivíduo por hectare ou c) espécies comuns, com mais de um indivíduo por hectare. De acordo com essa classificação $95,4 \%$ das espécies amostradas no presente trabalho são classificadas como raras, $1,5 \%$ intermediárias e 3,1\% comuns. O conceito de espécie rara tem sido usado para indicar as espécies que ocorrem com baixa densidade populacional em levantamentos estruturais. No entanto, essas espécies podem não ser necessariamente raras, mas sim apresentarem baixa densidade devido a alguns fatores relacionados aos erros de amostragem no inventário florestal. Durigan et al. (2000), comenta que entre esses fatores estão o tamanho da área amostral, as restrições estabelecidas nos levantamentos estruturais e o padrão de distribuição das espécies amostradas.

Segundo Ter Steege et al. (2013) milhares de espécies arbóreas na Amazônia são endêmicas e com alto risco de serem extintas antes mesmo de serem descritas taxonomicamente. As 5800 espécies mais raras, são suficientes para classificar aqueles que são endêmicas como globalmente ameaçadas. Juntos, esses 
táxons ( $36 \%$ das espécies mais raras) representam apenas 0,0003\% do total de árvores na Amazônia.

\section{Parâmetros estruturais, características tecnológicas e usos da madeira das principais espécies}

Entre as espécies levantadas, Eschweilera sp. (matamatá) foi a mais importante, com um Índice de Valor de Cobertura (IVC) de 32,62 e com 283 (12,90\%) indivíduos encontrados na área. Quanto ao mercado doméstico, em 2014 a tora do matamatá foi vendida por R\$159,00 por metro cúbico (SEMA, 2014). 0 matamatá produz uma "envira" muito forte e com diversos usos no meio rural e para a fabricação de móveis e artigos domésticos. Possui densidade básica de $0,81 \mathrm{~g} \cdot \mathrm{cm}^{-3}$, rápida secagem, com tendência a rachadura moderada e tendência ao torcimento e ao arqueamento forte. Os frutos e sementes de matamatá têm grande importância ecológica por atraírem fauna (ARAÚJO, 2000).

Manilkara amazonica (maparajuba) é a segunda espécie mais importante, com IVC de 19,36 e 144 (10\%) árvores. A espécie é comercializada a $\mathrm{R} \$ 219,00$ por metro cúbico de madeira em tora. A madeira de maparajuba possui densidade básica de $0,83 \mathrm{~g}_{\mathrm{cm}} \mathrm{cm}^{-3}$ e secagem natural em sete dias, com tendência moderada a rachaduras, a torcimento forte e a encanoamento médio. Tem indicação para uso a construção pesada e leve, embarcações, torneados chapas e instrumentos musicais. Assim como Eschweilera sp., M. amazonica é importante para a alimentação de animais silvestres (ARAÚJO, 2000)

Hymenaea courbaril (jatobá), mesmo não estando entre as cinco espécies mais abundantes neste estudo, apresentou indivíduos de grande porte, o que resultou no maior volume amostrado $\left(5,115 \mathrm{~m}^{3} \cdot \mathrm{ha}^{-1}\right.$, Tabela 1). A espécie contribuiu com $2,43 \%$ das árvores encontradas na área e teve área basal de $0,438 \mathrm{~m}^{2}$.ha 1. A madeira de H. courbaril vem sendo negociada a $\mathrm{R} \$$ 194,00 o metro cúbico. A espécie possui madeira pesada, ( $890 \mathrm{~kg} \cdot \mathrm{m}^{-3}$ a $12 \%$ de umidade ou $1240 \mathrm{~kg} \cdot \mathrm{m}^{-3}$ quando verde). A madeira tem textura média, secagem rápida e sem problemas de rachadura ou empenamento (SOUZA et al., 1997). Seu uso principal é na construção civil, molduras, móveis, cabos para ferramentas, materiais esportivos e musicais e dormentes. Além disso, H. courbaril produz frutos grandes, o que atrai a fauna silvestre (SOUZA et al., 1997; SUDAM, 1979; LOUREIRO, 1979; ARAÚJO, 2000). Segundo Nascimento et al. (2011), H. courbaril tem vasta distribuição geográfica e habilidade de se desenvolver em diferentes ambientes, pois a espécie tem baixas exigências nutricionais e hídricas. Estas características fazem do jatobá uma espécie com grande potencial silvicultural para plantios comerciais (PAIVA, 2003; SCHWARTZ et al., 2013).

Manilkara huberi (maçaranduba) apresentou 2,721 $\mathrm{m}^{3} . \mathrm{ha}^{-1}$. A madeira de M. huberi, negociada a $\mathrm{R} \$$ 193,00 o metro cúbico, é excelente para a construção civil e naval. Resiste ao ataque de fungos apodrecedores e é fácil de serrar, laminar, tornear, colar e parafusar (SOUZA et al., 1997). A madeira é pesada (1000 kg.m $\mathrm{m}^{-3}$ a $12 \%$ de umidade e $1260 \mathrm{~kg} \cdot \mathrm{m}^{-3}$ quando verde), de secagem rápida em estufa e de tendência moderada a rachaduras (SOUZA et al., 1997; SUDAM, 1979; ARAÚJO, 2000).

A espécie Goupia glabra (cupiúba) ocorre com abundância em toda a Amazônia brasileira, Guiana, Colômbia, Venezuela, Guiana Francesa, Peru e Suriname (LOUREIRO et al., 1979). Na área de estudo ocorreu com 0,809 indivíduo.ha- ${ }^{-1}$, área basal de $0,41 \mathrm{~m}^{2}$.ha ${ }^{-1}$ e volume de $2,577 \mathrm{~m}^{3} \cdot \mathrm{ha}^{-1}$. A espécie vem sendo vendida a $R \$ 156,00$ por metro cúbico e tem aplicação na construção civil e naval. A sua madeira é facilmente 
trabalhável, mas não é adequada para fabricação de compensados por apresentar rachaduras na tora (SOUZA et al., 1997). Segundo Souza et al. (1997), a madeira de G. glabra possui densidade média (840 kg.m ${ }^{-3}$ a $12 \%$ de umidade e $1130 \mathrm{~kg} \cdot \mathrm{m}^{-3}$ quando verde) (SUDAM, 1979; SOUZA et al., 1997; ARAÚJO, 2000).

Na sexta colocação em hierarquia, com 30 indivíduos, ficou Lecythis usitata (sapucaia), com um volume comercial de $1,923 \mathrm{~m}^{3} \cdot \mathrm{ha}^{-1}$ na área de estudo. A sua madeira é comercializada na região devido à grande utilidade na construção civil, naval, móveis e artigos domésticos. Além disso, os frutos de L. usitata são usados na alimentação humana também atraem animais silvestres (ARAÚJO, 2000).

Outras espécies registradas neste estudo como Brosimum parinarioides, Dipteryx odorata, Protium puncticulatum, Piptadenia suaveolens e Lecythis lurida apresentam alto valor comercial (R\$154,00; R\$ 208,00; $R \$ 154,00 ; R \$ 155,00$ e $R \$ 147,00$; respectivamente) e diversas utilidades como lenha e carvão, embarcações, carpintaria, dormentes, cavacos para cobrir casas, estacas, moirões, vigamentos e tacos para assoalhos (ARAÚJO, 2000).

Os resultados sugerem que as espécies Sterculia pilosa, Piptadenia suaveolens, Anacardium giganteum e Cordia alliodora devem ser mantidas na área com as mesmas proporções em relação à abundância e frequência. Além disso, deverão ser consideradas quanto à sua importância ecológica nos planos de exploração em áreas com características semelhantes à área estudada neste trabalho. As espécies Pithecelobium racemosum, Sterculia pilosa, Dipteryx odorata, Goupia glabra, Parkia gigantocarpa, Parkia nitida, Parkia multijulga, Cordia alliodora, Simaruba amara, Schefflera morotoni, Parkia pendula, Jacaranda copaia, Euxylophora paraensis, Caryocar microcarpum, Caryocar globum e Bagassa guianensis, que estão relacionadas entre as ecologicamente mais importantes na área de estudo, podem ainda ser utilizadas na recuperação de áreas alteradas (PINHEIRO et al., 2007).

\section{Comercialização local para a madeira}

No período entre 01/01/2014 a 25/05/2014 a extração e movimentação de toras de madeira nativa, classificado por espécie vegetal, indicando a quantidade de carga, volume em metros cúbicos e o valor comercializado, chegaram a 1.007.544,5133 $\mathrm{m}^{3}$ com um valor total comercializado de $\mathrm{R} \$ 182.117 .661,23$ (SEMA, 2014). Neste estudo, as espécies com maiores valores comerciais por metro cúbico de madeira em toda foram Cedrela odorata ( $\mathrm{R} \$ 599,00)$, Tabebuia serratifolia ( $\mathrm{R} \$ 427,00)$, Cordia goeldiana $(\mathrm{R} \$ 424,00)$, Dipteryx odorata (R\$ 208,00) e Bowdichia nitida (R\$ 202,00). As espécies Dinizia excelsa, Cordia alliodora, Diplotropis purpurea e Bagassa guianensis tiveram valor médio de $\mathrm{R} \$ 197,00$ enquanto que Ceiba pentandra, Anacardium giganteum, Jacaranda copaia, Qualea lancifolia e Enterolobium maximum foram vendidas abaixo de $R \$ 150,00$ por metro cúbico de madeira em tora.

\section{Comercialização local para produtos não-madeireiros}

Assim como as frutas obtidas do extrativismo, os produtos medicinais vêm tendo maior aceitação nos centros urbanos (PINHEIRO et al., 2009). Óleos vegetais, especialmente o de copaíba e o de andiroba, são bastante difundidos na região Norte, sendo comercializados para outras regiões do Brasil na forma de 
sabonetes e xampus (PINTO et al., 2003). Muitas espécies amostradas neste estudo também vêm tendo grande importância medicinal (Tabela 2).

Tabela 2: Indicação de uso não madeiráveis de algumas espécies arbóreas que ocorrem na fazenda Rio Capim, Paragominas, PA.

\begin{tabular}{|c|c|}
\hline NOME COMUM & UTILIZAÇÃO \\
\hline $\begin{array}{l}\text { Amapá-doce } \\
\text { Brosimum } \\
\text { parinarioides }\end{array}$ & O látex é aplicado contra doenças pulmonares. \\
\hline $\begin{array}{l}\text { Amesclão } \\
\text { Trattinickia } \\
\text { burseraefolia }\end{array}$ & A semente é utilizada no tratamento da asma. \\
\hline $\begin{array}{l}\text { Andiroba } \\
\text { Carapa guianensis }\end{array}$ & O óleo é aplicado em inchaços e no tratamento da tosse, pode ser usado na fabricação de vela e sabão. \\
\hline $\begin{array}{l}\text { Angico } \\
\text { Piptadenia } \\
\text { suaveolens }\end{array}$ & A casca é usada como chá para depurativo de sangue, e a resina para baques e reumatismos. \\
\hline $\begin{array}{l}\text { Caju-açu } \\
\text { Anacardium } \\
\text { giganteum }\end{array}$ & O fruto é usado na alimentação. \\
\hline $\begin{array}{l}\text { Copaíba } \\
\text { Copaifera multijuga }\end{array}$ & O óleo é utilizado na iluminação caseira e tem uso medicinal contra inflamação. \\
\hline $\begin{array}{l}\text { Cumaru } \\
\text { Dipteryx odorata }\end{array}$ & $\begin{array}{l}\text { As amêndoas dos frutos são aromáticas e usadas em perfumarias e na composição de licores, vermutes e } \\
\text { xaropes; a tintura das cascas é antiespasmódica e tônica. }\end{array}$ \\
\hline $\begin{array}{l}\text { Cupiuba } \\
\text { Goupia glabra }\end{array}$ & As sementes são utilizadas para tratamento de sinusite. \\
\hline $\begin{array}{l}\text { Jatobá } \\
\text { Hymenaea courbaril }\end{array}$ & Utilizada como vermífuga tônica, adstringente, balsâmica. \\
\hline $\begin{array}{l}\text { Mururé } \\
\text { Brosimum obovata }\end{array}$ & O chá da casca é usado como antianêmico. \\
\hline $\begin{array}{l}\text { Parapará } \\
\text { Jacaranda copaia }\end{array}$ & A semente e a casca são utilizadas para desinfetar e defumar ambientes. \\
\hline $\begin{array}{l}\text { Sapucaia } \\
\text { Lecythis usitata }\end{array}$ & Possui óleo comestível. A casca é tônica e diurética. \\
\hline $\begin{array}{l}\text { Uxi } \\
\text { Endopleura uchi }\end{array}$ & Os frutos são utilizados na alimentação. \\
\hline
\end{tabular}

Na cidade de Belém/PA, por exemplo, os produtos não madeireiros são comercializados em feiras livres e em lojas especializadas em produtos naturais (SHANLEY et al., 2003). Já em Moju e em Abaetetuba, no interior do Pará, esses produtos são negociados em menor escala em algumas barracas de feira livre. A maioria dos produtos medicinais comercializados no interior do Pará é obtida pelos próprios feirantes enquanto que na capital (Belém) os produtos são adquiridos de agricultores de município vizinhos (PINHEIRO et al., 2009).

\section{CONCLUSÕES}

A área estudada na fazenda Rio Capim apresenta uma composição florística de alto valor ecológico e econômico. A estrutura da floresta permite que a mesma seja manejada para produção de madeira e de outros produtos florestais, observando os padrões de sustentabilidade econômica, ecológica e social. Quanto à estrutura da floresta estudada, as famílias Leguminosae, Lecythidaceae e Sapotaceae foram as que tiveram maiores número de espécies e indivíduos. Da mesma forma, algumas espécies se sobressaíram na estrutura da floresta como Eschweilera sp. (matamatá), Manilkara amazonica (maparajuba), Hymenaea courbaril (jatobá), Manilkara huberi (maçaranduba), Goupia glabra (cupiúba) e Lecythis usitata (sapucaia). Outras 13 
espécies foram identificadas como importantes para produtos não madeireiros (medicinais ou para a produção de frutos). Considerando a importância dessas famílias e dessas espécies na estrutura da floresta, assim como na economia, o manejo a ser executado na área de estudo deve visar a sua conservação, utilizando práticas adequadas de exploração florestal e de tratos silviculturais.

Atualmente, os projetos de manejo florestal no estado do Pará estão aprimorando as máquinas e as técnicas utilizadas, resultando numa maior eficiência na exploração e no processamento da madeira. A maioria das espécies ecologicamente mais importantes em florestas vizinhas, com estruturas semelhantes a esta estudada, poderão ser utilizadas sob regime de rendimento sustentável, com o mínimo de alteração em sua ecologia, considerando a sua consistência estrutural, essas espécies possivelmente serão perpetuadas na área. Por fim, as recomendações aqui apresentadas podem ser aplicadas em outras florestas tropicais com estrutura semelhante à da floresta estudada neste trabalho.

\section{REFERÊNCIAS}

ALVES, J. C. Z.; MIRANDA, I. S.. Analise da estrutura de comunidades arbóreas de uma floresta amazônica de terra firme aplicado ao manejo florestal. Acta Amazônica, v.38, n.4, p.657-666, 2008.

ARAÚJO, H. J. B.; SILVA, I. G.. Lista de espécies florestais do Acre: ocorrência com base em inventários florestais. Rio Branco: Embrapa Acre, 2000.

BARROS, A. V.; BARROS, P. L. C.; SILVA, L. C. B.. Estudo da diversidade de espécies de uma floresta situado em CuruáUna - Pará. Revista de Ciências Agrárias, Belém, n.34, p.4965, 2000.

BENTES-GAMA, M. M.. Estrutura, valoração e opções de manejo sustentado para uma floresta de várzea na Amazônia. Dissertação (Mestrado em Engenharia Florestal) Universidade Federal de Lavras, Lavras, 2000.

BRASIL. Ministério do Meio Ambiente. Instrução Normativa N. 5 de 11 de dezembro de 2006. Brasília: MMA, 2006.

CARVALHO, J. O. P.. Dinâmica de florestas naturais e sua implicação para o manejo florestal. In: SIMPÓSIO SILVICULTURA NA AMAZÔNIA ORIENTAL: CONTRIBUIÇÕES DO PROJETO EMBRAPA/DFID, 1. Anais. Belém: EMBRAPACPATU/DFID, 1999. p.174-179.

CARVALHO, J. O. P.; ARAÚJO, S. M.; CARVALHO, M. S. P.. Estrutura horizontal de uma floresta secundária no planalto do Tapajós em Belterra, Pará. In: SIMPÓSIO DO TRÓPICO ÚMIDO, 1. Anais. Belém: EMBRAPA/CPATU, 1986. p.207215.

COSTA, D. H. M.; CARVALHO, J. O. P.; SILVA, J. N. M.. Dinâmica da composição florística após a colheita de madeira em uma área de terra firme na Floresta Nacional do Tapajós (PA). Revista de Ciências Agrárias, Belém, n.38, p.67-80, 2002.

DURIGAN, G.; RODRIGUES, R. R.; SCHIAVINI, I.. A heterogeneidade ambiental definindo a metodologia de amostragem da floresta ciliar. In: RODRIGUES, R. R.; LEITÃO FILHO, H. F.. Matas ciliares: conservação e recuperação. EDUSP, São Paulo. 2000. p.159-167.

DYKSTRA, D. P.. Has reduced-impact logging outlived its usefulness?. Journal of Tropical Forest Science, v.24, p.1-4, 2012.
FRANCEZ, L. M. B.; CARVALHO, J. O. P.; JARDIM, F. C. S. QUANZ, B.; PINHEIRO, K. A. O.. Efeito de duas intensidades de colheitas de madeira na estrutura de uma floresta natural na região de Paragominas, Pará. Acta Amazônica, v.39, n.4, p.851-864.

FRANCEZ, L. M. B.; CARVALHO, J. O. P.; JARDIM, F. C. S.. Mudanças ocorridas na composição florística em decorrência da exploração florestal em uma área de floresta de Terra firme na região de Paragominas, PA. Acta Amazônica, v.37, n.2, p.219-228, 2007.

KAGEYAMA, P.; GANDARA, F. B.. Recuperação de áreas ciliares. In: RODRIGUES, R. R.; LEITÃO FILHO, H. F.. Matas ciliares: conservação e recuperação. São Paulo: EDUSP, 2000. p.249-269.

LAWSON, S.; MACFAUL, L.. Illegal logging and related trade Chatham House: London. 2010.

LEAL, G. L. R.. Paragominas: a realidade do pioneirismo. Belém: Alves, 2000.

LOUREIRO, A. A.. Essências madeireiras da Amazônia. Manaus: INPA, 1979.

MAUÉS, M. M.. Estratégias reprodutivas de espécies arbóreas e sua importância para o manejo e conservação florestal: Floresta Nacional do Tapajós (Belterra/PA). Tese (Doutorado) - Universidade de Brasília, Brasília, 2006.

MILLIKEN, W.. Structure and composition of one hectare of Central Amazon terra firm forest. Biotropica, v.30, n.4, p.2738, 1998.

MORAIS-CRUIA, A. P. O.; VEIGA, J. B.; LUDONINO, R. M. R.; SIMÃO NETO, M.; TOURRAND, J. F.. Caracterização dos sistemas de produção da agricultura familiar de Paragominas-PA: a pecuária e propostas de desenvolvimento. Belém: Embrapa Amazônia Oriental, 1999.

MYERS, N.. Tropical forests: The main deforestation fonts. Env. Conser., n.20, p.9-16, 1993

NASCIMENTO, H. H. C.; NOGUEIRA, R. J. M. C.; SILVA, E. C.; SILVA, M. A.. Análise do crescimento de mudas de jatobá (Hymenaea courbaril I.) em diferentes níveis de água no solo. Revista Árvore, Viçosa, v.35, n.3, p.617-626, 2011. 
OLIVER, C. D.; LARSON, B. C.. Forest stand dynamics New York: John Wiley \& Sons, 1996.

PAIVA, H. N.; VITAL, B. R.. Escolha da espécie florestal. Viçosa: Universidade Federal de Viçosa, 2003.

PINHEIRO, K. A. O.; CARVALHO, J. O. P.; QUANZ, B.; FRANCEZ, L. M. B.; SCHWARTZ, G.. Fitossociologia de uma área de preservação permanente no leste da Amazônia: indicação de espécies para recuperação de áreas alteradas. Revista Floresta, Curitiba, v.37, n.2, 2007.

PINHEIRO, K. A. O.; SCHWARTZ, G.. Aspectos socioeconômicos da comercialização de produtos agrícolas e florestais em sete comunidades rurais no alto moju, estado do Pará. Amazônia: Ci. \& Desenvolvimento, Belém, v.5, n.9, 2009.

PINTO, A. A. C.; MADURO, C. B.. Produtos e subprodutos da medicina popular comercializados na cidade de Boa Vista, Roraima. Acta Amazônica, Manaus, v.33, n.2, p.281-289, 2003.

PINTO, A. C. M.; SOUZA, A. L.; SOUZA, A. P.; MACHADO, C. C.; MINETTE, L. J.; VALE, A. B.. Analise de danos de colheita de madeira em floresta tropical úmida sob regime de manejo florestal sustentado na Amazônia Ocidental. Revista Árvore, v.26, n.4, p.459-466, 2002.

MERONA, J.; PRANCE, G. T.; HUTCHINGS, R. W.; SILVA, M. F. da; RODRÍGUEZ, W. A.; VENLING, M. E.. Preliminary results of a large-scale tree inventory of upland rain forest in the central Amazon. Acta Amazônica, v.22, n.4, p.485-492, 1992.

RODRIGUEZ, L. C. E.. Monitoramento florestal: iniciativas, definições e recomendações. IPEF, v.12, n.31, p.9-21, 1998.

SABOGAL, C.; LENTINE, M.; PORKONY, B.; SILVA, J. N. M.; ZWEEDE, Z.; VERRISIMO, A.; BOSCOLO, M.. Manejo florestal empresarial na Amazônia Brasileira. Belém: CIFOR, 2006.

SALOMÃO, R. P.. Uso de parcela permanente para estudo da vegetação da floresta tropical úmida, Município de Marabá, Pará. Boletim do Museu Emílio Goeldi, v.4, n.2, p.195-233, 1991.

SANTANA, A. C.; SANTOS, M. A. S.; SANTANA, Á. L.; YARED, J. A. G.. O valor econômico da extração manejada de madeira no baixo amazonas, estado do Pará. Revista Árvore, Viçosa, v.36, n.3, p.527-536, 2012

SCHWARTZ, G.; LOPES, J. C. A.; MOHREN, G. M. J.; PEÑACLAROS, M.. Post-harvesting silvicultural treatments in logging gaps: A comparison between enrichment planting and tending of natural regeneration. Forest Ecology and Management, Amsterdã, v.293, p.57-64, 2013.
SCHWARTZ, G.; PEÑA-CLAROS, M.; LOPES, J. C. A.; MOHREN, G. M. J.; KANASHIRO, M.. Mid-term effects of reducedimpact logging on the regeneration of seven tree commercial species in the Eastern Amazon. Forest Ecology and Management, Amsterdã, v.274, p.116-125, 2012

SHANLEY, P.; LUZ, L.. The impacts of forest degradation on medicinal plant use and implications for health care in Eastern Amazonia. Bioscience, Madison, v.53, n.6, p.573584, 2003.

SILVA, A. S. L.; LISBOA, P. L. B.; MACIEL, U. N.. Diversidade florística e estrutura em floresta densa da bacia do Rio Juruá. Boletim do Museu Paraense Emílio Goeldi, v.8, n.2, p.203-258, 1992.

SILVA, J. N. M.. Manejo Florestal. Brasília: Embrapa SPI, 1996.

SILVA, K. E. F.; MATOS, D. A.; FERREIRA, M. M.. Composição florística e fitossociologia de espécies arbóreas do Parque Fenológico da Embrapa Amazônia Ocidental. Acta Amazônica, v.38, n.2, p.213-222, 2008.

SILVA, R. C.. Contribuição do levantamento de solo e caracterização dos sistemas naturais e ambientais na região de Paragominas, Estado de Pará. Dissertação (Mestrado em Agronomia) - Faculdade de Ciências Agrárias do Pará, Belém, 1997.

SOMBROEK, W. G.. Soil of the Amazon region. In: SIOLI, H. The Amazon Basin: Landscape Ecology and Hydrology of a Mighty River. 1986. p.122-135.

SOUZA, M. H.; MAGLIANO, M. M.; CAMARGOS, J. A. A.; SOUZA, M. R.. Madeiras tropicais brasileiras. Brasília: IBAMA, 1997.

SUDAM. Departamento de Recursos Naturais. Centro de Tecnologia Madeireira. Pesquisa e Informações dobre espécies florestais da Amazônia. Belém: SUDAM, 1979.

TER STEEGE, H. et al. Hyperdominance in the Amazonian tree flora. Science, v.342, p.325-334, 2013.

VALE, A. B.. Progresso no manejo de matas naturais. Revista Floresta, Curitiba, n.4, p.69-74. 1972.

VELOSO, H. P.; RANGEL FILHO, A. L. R.; LIMA, J. C. A. Classificação da vegetação brasileira, adaptada a um sistema universal. Rio de Janeiro: IBGE, 1991.

WATRIN, O. S.; ROCHA, A. M. A.. Levantamento da vegetação natural e do uso da terra no município de Paragominas (PA) utilizando imagens TM/ LANDSAT. Belém: EMBRAPA-CPATU, 1992.

WHITMORE, T. C.. An introduction to tropical rain forests. Oxford: Press, 1990.

A CBPC - Companhia Brasileira de Produção Científica (CNPJ: 11.221.422/0001-03) detém os direitos materiais desta publicação. Os direitos referem-se à publicação do trabalho em qualquer parte do mundo, incluindo os direitos às renovações, expansões e disseminações da contribuição, bem como outros direitos subsidiários. Todos os trabalhos publicados eletronicamente poderão posteriormente ser publicados em coletâneas impressas sob coordenação da Sustenere Publishing da Companhia Brasileira de Produção Científica e seus parceiros autorizados. Os (as) autores (as) preservam os direitos autorais, mas não têm permissão para a publicação da contribuição em outro meio, impresso ou digital, em português ou em tradução. 